\title{
A MATHEMATICAL PROBLEM FROM DETONATION THEORY*
}

\author{
BY \\ WILDON FICKETT
}

Los Alamos National Laboratory, Los Alamos, New Mexico

\begin{abstract}
A simplified abstraction of a partial-differential-equation problem which appears in the study of small perturbations on a detonation wave is studied. The interesting feature of this problem is that the function representing the unknown values of the dependent variable on one boundary appears as a source term in the partial differential equation. This unknown boundary function turns out to be the solution of an ordinary differential-difference equation. We study the properties of this differential-difference equation, and also present some representative solutions of the complete problem.
\end{abstract}

I. Introduction. A detonation wave, Fig. 1, consists of a shock followed by a zone of chemical reaction [1]. The shock is a discontinuous jump in state. After passage through the shock, a particle of explosive finds itself in a brand new environment-a temperature of several thousand degrees and a pressure of several hundred thousand atmospheres. Chemical reaction begins immediately, and proceeds to completion in the narrow reaction zone that follows the shock. Figure 2 is an $x-t$ (distance-time) diagram of the detonation. In the steady detonation (dashed lines) the path of the shock and the locus of complete reaction are straight lines.

The behavior of small perturbations applied to the steady solution is treated in [2]. A perturbation applied to the steady detonation produces a variation in the shock velocity, as indicated by the solid line in the figure. (The locus of complete reaction is in general also perturbed, but this is not shown in the figure.) The problem of describing the evolution of the perturbation is a difficult one because the shock is a floating boundary whose location is unknown. It can be simplified by transforming to an accelerated frame attached to the perturbed shock. In this frame the shock path is a straight line, and (under certain restrictions on the reaction rate) the problem space becomes rectangular. The price paid for this simplification in the geometry of the problem is the appearance of the unknown shock state in the coefficients of the governing partial differential equations. After linearization, the unknown shock state appears as a source term.

*Received March 20, 1987.

(C)1988 Brown University 


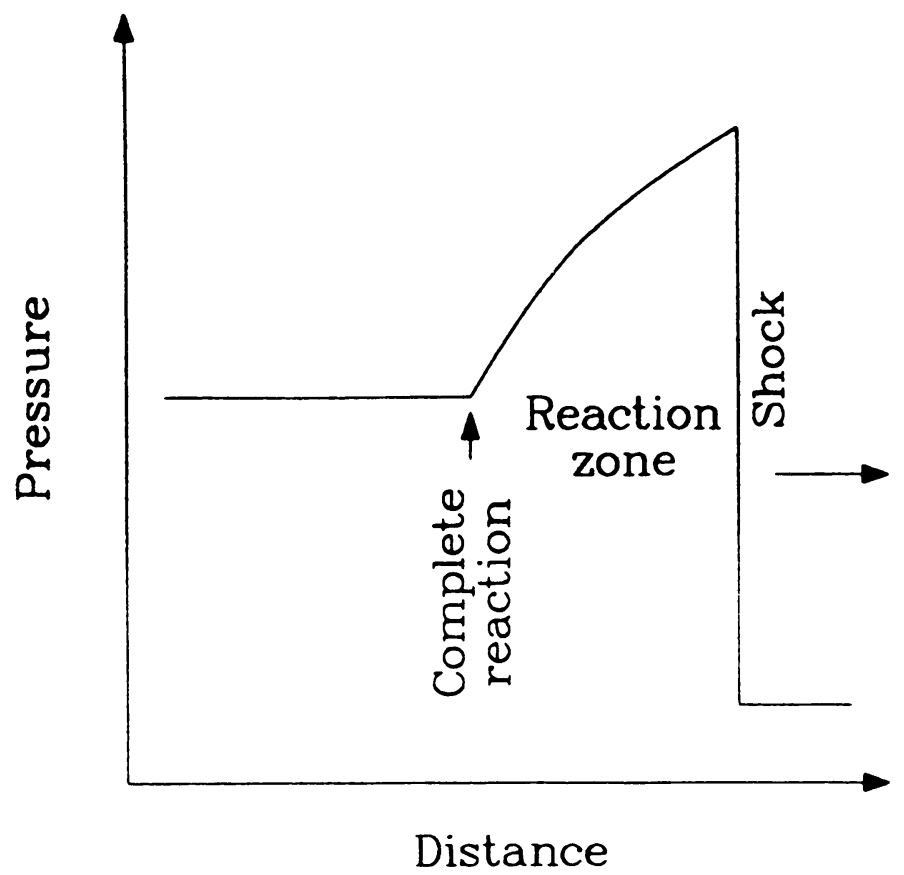

Fig. 1. Profile of a detonation wave.

In this paper we consider the model problem

$$
\begin{aligned}
u_{t}+u_{x} & =-u+u(1, t), \\
0 & \leq x \leq 1, \\
0 & \leq t, \\
u & =u_{i}(x) \quad \text { on } t=0, \\
u & =u_{b}(t) \quad \text { on } x=0,
\end{aligned}
$$

a simplified abstraction which we have constructed to illustrate the interesting mathematical features of the problem described above.

The problem space for (1.1) is shown in Fig. 3. (The choice of independent variables here corresponds to a ninety degree rotation from the rectangular space of the original problem, making $t$ the evolution variable instead of $x$.) The dependent variable $u$ is the perturbation on the steady solution. The right boundary $x=1$ is the (perturbed) shock path, and the left boundary $x=0$ is the end of the reaction zone (the locus of complete reaction). The initial data $u_{i}(x)$ on $t=0$ and the boundary data $u_{b}(t)$ on $x=0$ are the applied perturbations. In the original space, Fig. 2, the perturbation is applied along the rear boundary $x=0$. The segment within the reaction zone becomes $u_{i}(x)$ in the space of Fig. 3, and the remainder, transferred to the end of the reaction zone, becomes $u_{b}(t)$. See [2] for a more detailed discussion.

The governing equation (1.1) is hyperbolic. On the left we have the wave operator $u_{t}+u_{x}$. Its characteristic paths are indicated by the diagonal lines of the figure, along 


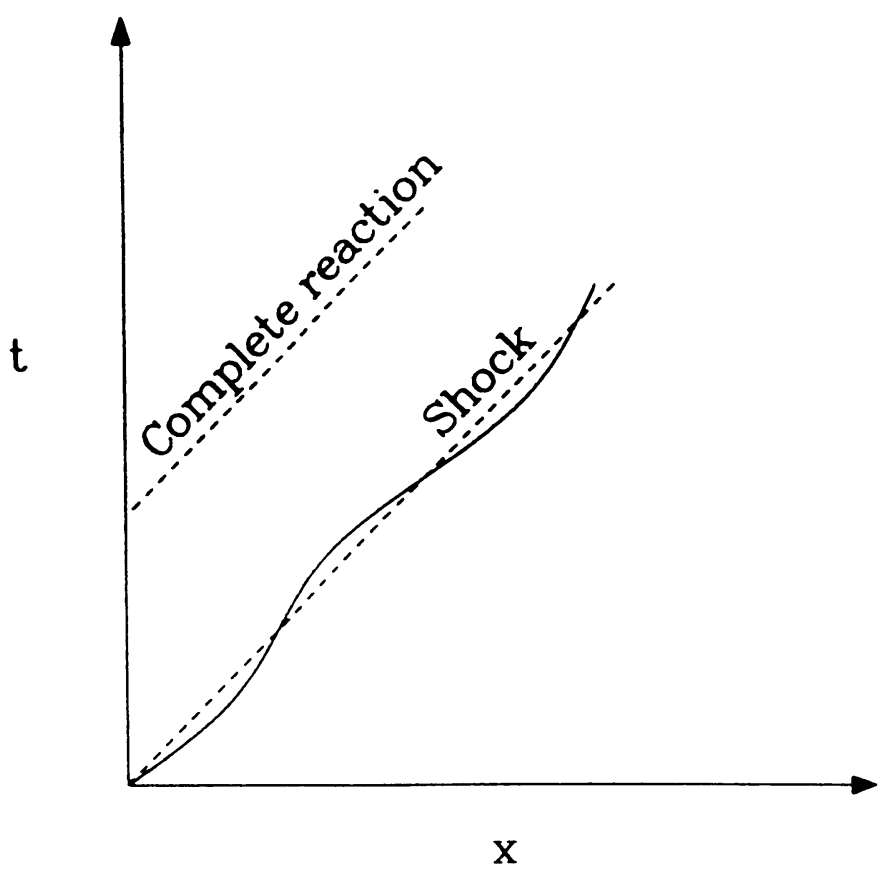

Fig. 2. Detonation, $x-t$ diagram. (---) steady, (-) perturbed.

which perturbations from the lower and left boundaries travel to the shock. On the right are two source terms. The first term, $-u$, is related, somewhat indirectly, to the heat released by the chemical reaction. The second term, $u(1, t)$, represents the effect of introducing the unknown shock velocity into the governing equations by the frame transformation. Because the shock is the trigger which starts the chemical reaction, its position is an important element of the problem. This term takes this triggering effect into account.

Our object is to elucidate the behavior of the system (1.1), that is, to understand how the system responds to an applied perturbation. The main results are:

1. a differential-difference equation for the unknown right-boundary function, analysis of which gives the natural frequencies of the system;

2. a small collection of particular solutions of (1.1), chosen to illustrate the nature of the system's response to perturbations.

A method similar to that described here has been previously employed in studies of electrical oscillations in transmission lines [3], [4]. A pair of partial differential equations with a nonlinear boundary condition is reduced to a differential-difference equation along a boundary.

In Sec. II, a formal solution of (1.1) leads to a differential-difference equation for the unknown right-boundary function $u(1, t)$. This differential-difference equation is studied in Secs. III and IV. The collection of particular solutions of (1.1) is obtained 


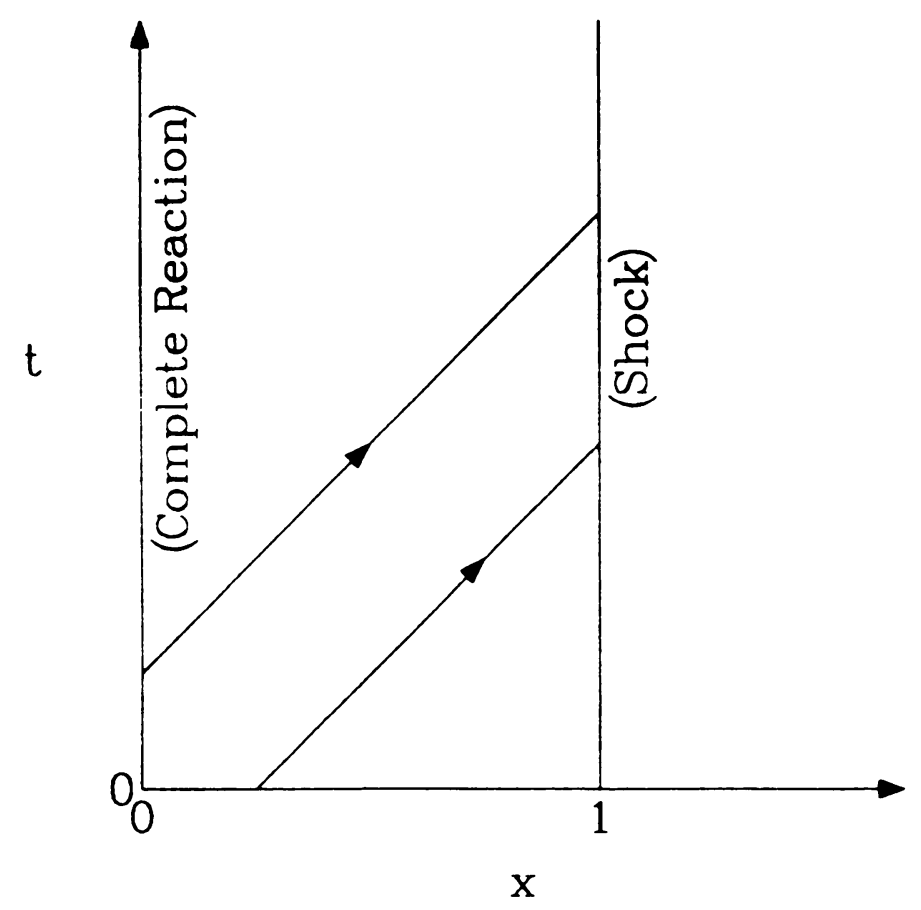

FIG. 3. The problem space.

in Sec. V, and summarized in Sec. VI. At the end of Sec. II, with the formal solution in hand, we give a more detailed outline of our subsequent plan of attack.

II. Solution. We solve (1.1) by the method of characteristics. Along the characteristic curves in $x$ - $t$ the partial differential equation (1.1) becomes an ordinary differential equation. To find these curves, consider the directional derivative of $u$ along a curve $C$ defined by $(d x / d t)_{C}=f(t)$, that is, the directional derivative $(d u / d t)_{C}=u_{t}+f(t) u_{x}$. If we choose $f(t)=1$, we get the wave operator on the left side of (1.1). We can then write (1.1) in the so-called characteristic form

$$
d u / d t=-u+u_{1} \quad \text { on } d x / d t=1,
$$

where we have abbreviated $u(1, t)$ to

$$
u_{1}=u_{1}(t) \equiv u(1, t)
$$

The characteristic curves are indicated in the figures by diagonal lines marked with arrows.

The problem space divides itself naturally into two regions, which we designate as I and II, as shown in Fig. 4. The dividing line is the characteristic curve through the lower left corner. In region I we obtain an explicit solution involving an integral over the initial-data function $u_{i}(x)$. In region II, a formal solution by the method of characteristics leads to a differential-difference equation for the shock state $u_{1}(t)$. 


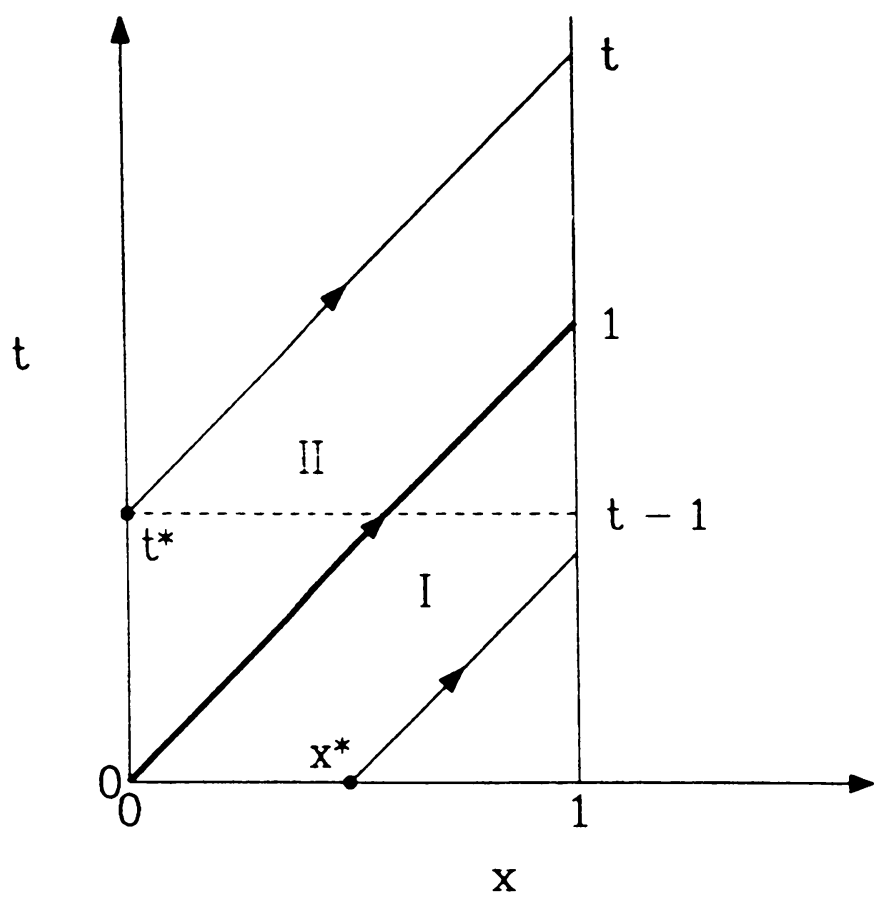

FIG. 4. Regions of the problem space and arguments of the differentialdifference equation (2.10).

The initial data for this differential-difference equation are the values of $u_{1}$ on the unit interval $[0,1]$ (of the right boundary) obtained from the region-I solution.

Region I. Consider the characteristic path, $t=x-x^{*}$, through a point $x^{*}$ on the lower boundary of Fig. 4. On this path, we have from (2.1) the ordinary differential equation

$$
\begin{aligned}
d u / d t & =-u+u_{1} \quad \text { on } t=x-x^{*}, \\
u & =u_{i}\left(x^{*}\right) \text { at } t=0 .
\end{aligned}
$$

Integration gives

$$
u\left(t ; x^{*}\right)=e^{-t}\left[u_{i}\left(x^{*}\right)+\int_{0}^{t} u_{1}\left(t^{\prime}\right) e^{t^{\prime}} d t^{\prime}\right] .
$$

This is the formal solution in region $\mathrm{I}$, but the right-boundary function $u_{1}(t)$ is still unknown. To obtain an equation for $u_{1}(t)$ we evaluate this result on the right boundary $x=1$. On the left side of the equation, $u\left(t ; x^{*}\right)$ becomes $u_{1}(t)$. On the right side, $x^{*}$ becomes $1-t$. We have then

$$
u_{1}(t)=e^{-t}\left[u_{i}(1-t)+\int_{0}^{t} u_{1}\left(t^{\prime}\right) e^{t^{\prime}} d t^{\prime}\right], \quad 0 \leq t \leq 1,
$$

a Volterra integral equation for $u_{1}(t)$. We convert this to a differential equation by differentiating with respect to $t$. We find for $u_{1}(t)$ the ordinary differential equation 
and initial condition

$$
\begin{aligned}
& u_{1}^{\prime}(t)=-e^{-t} u_{i}^{\prime}(1-t) \\
& u_{1}(0)=u_{i}(1)
\end{aligned}
$$

Integration gives

$$
u_{1}(t)=u_{i}(1)-\int_{0}^{t} u_{i}^{\prime}\left(1-t^{\prime}\right) e^{-t^{\prime}} d t^{\prime}, \quad 0 \leq t \leq 1 .
$$

We have thus found the unknown right-boundary function $u_{1}(t)$ in region $\mathrm{I}$. The solution $u(x, t)$ in region $\mathrm{I}$ is then given in parametric form by (2.3). (For given $x$ and $t$, we can find the parameter $x^{*}$ from $x^{*}=x-t$.)

Region II. We proceed in similar fashion here. On the characteristic path through a point $t^{*}$ on the left boundary (Fig. 4) we have

$$
\begin{aligned}
& d u / d t=-u+u_{1}, \quad \text { on } t=x+t^{*}, \\
& u=u_{b}\left(t^{*}\right) \quad \text { at } t=t^{*} \text {. }
\end{aligned}
$$

The formal solution in region II is

$$
u\left(t ; t^{*}\right)=e^{-t}\left[e^{t^{*}} u_{b}\left(t^{*}\right)+\int_{t^{*}}^{t} u_{1}\left(t^{\prime}\right) e^{t^{\prime}} d t^{\prime}\right], \quad t>x .
$$

Evaluating this on the right boundary $x=1$, where $t^{*}$ is $t-1$ and $u$ is $u_{1}$, we find

$$
u_{1}(t)=e^{-t}\left[e^{t-1} u_{b}(t-1)+\int_{t-1}^{t} u_{1}\left(t^{\prime}\right) e^{t^{\prime}} d t^{\prime}\right],
$$

an integral equation for $u_{1}(t)$. Differentiating this with respect to $t$ gives the desired differential-difference equation in region II (with its initial condition)

$$
\begin{aligned}
u_{1}^{\prime}(t)+a u_{1}(t-1) & =a\left[u_{b}(t-1)+u_{b}^{\prime}(t-1)\right], \\
a & \equiv e^{-1}, \\
u_{1}(t) & \text { given, on } 0 \leq t \leq 1 .
\end{aligned}
$$

This is one of our principal results. Figure 4 shows, for a given $t^{*}$, the characteristic path through $t^{*}$ and the corresponding arguments $t$ and $t-1$ of (2.10). As stated earlier, the initial data for this equation are values of $u_{1}(t)$ on the unit interval $[0,1]$, obtained from the solution in region I. We remark that the differential-difference equation (31) of [2] reduces to (2.10) for $\beta=1$ (which gives $\alpha=e^{-1}$ ).

Discontinuous solutions. We will want to consider later solutions having a discontinuity on the dividing line between regions I and II, generated by a discontinuity at the origin between the given initial data $u_{i}(t)$ and the boundary data $u_{b}(t)$. We set down here for later use expressions for $u$ on both sides of the dividing characteristic (see Fig. 4). Let $u_{-}$and $u_{+}$denote values of $u$ just below and just above the dividing characteristic. To find these we first calculate $u_{1}(t)$ from $u_{i}(t)$ over the range $0 \leq t<1$ from (2.6). Then we calculate $u_{-}$from (2.3) with $x^{*}=0$ and $u_{+}$ from $(2.8)$ with $t^{*}=0$. Omitting the explicit indication of the parameter $\left(x^{*}=0\right.$ or $\left.t^{*}=0\right)$, we can write $(2.3)$ or $(2.8)$ in the form

$$
u(t)=e^{-t}\left[u(0)+\int_{0}^{t} u_{1}\left(t^{\prime}\right) e^{t^{\prime}} d t^{\prime}\right] .
$$


The magnitude of the jump itself is independent of $u_{1}(t)$ because the integral in (2.11) cancels. We find

$$
u_{+}(t)-u_{-}(t)=e^{-t}\left[u_{+}(0)-u_{-}(0)\right]=e^{-t}\left[u_{b}(0)-u_{i}(0)\right] .
$$

Note that the size of the jump decreases exponentially with time.

A case we will use later is

$$
u_{i}(x)=1, \quad u_{b}(t)=0,
$$

for which

$$
\begin{aligned}
& u_{1}(t)=1, \quad 0 \leq t<1, \\
& u_{-}(t)=1, \\
& u_{+}(t)=1-e^{-t} .
\end{aligned}
$$

Summary and plan. We now have a formal solution to our problem. With certain continuity restrictions on its initial data, the linear first-order differential-difference equation $(2.10)$ for the unknown right-boundary function $u_{1}(t)$ in region II can be reduced to a quadrature in a manner similar to that for the linear first-order differential equation, see [5], Theorem (3.7). The resulting expression for $u_{1}(t)$ can then be substituted in the integrands of the solutions (2.3) and (2.8), giving the solution to the complete problem in the form of a double quadrature.

Our main object is to get a feel for the behavior of the system (1.1). We shall study first the properties of the differential-difference equation (2.10), obtaining from it the natural frequencies of the system in Sec. III, and power-series solutions (for certain initial conditions) which will be convenient for the numerical evaluation to follow in Sec. IV. With the right-boundary function in hand, we obtain in Sec. V several particular solutions of (1.1). In Sec. VI we summarize these in a conceptual framework which helps to illuminate the behavior of the system.

III. Natural frequencies. Differential-difference equations like (2.10) are discussed in [5]. The homogeneous part of $(2.10)$ is

$$
u_{1}^{\prime}(t)+a u_{1}(t-1)=0 .
$$

This equation has an exponential solution $e^{s t}, s$ complex, provided $s$ satisfies the eigenvalue condition

$$
G(s)=s+a e^{-s}=0,
$$

obtained by substituting $e^{s t}$ in (3.1). The function $G(s)$ is the reciprocal of the transfer function of the system. The general solution of (3.1) can be constructed as an eigenfunction expansion of the form

$$
u_{1}(t)=\sum_{i=1}^{\infty} c_{i} e^{s_{i} t},
$$

where the $s_{i}$ are the roots of the complex function $G(s)$, and the coefficients $c_{i}$ are determined by the initial data. Thus the roots of $G(s)$ (poles of the transfer functions) give the natural frequencies and decay rates of the system. 
We now discuss these roots. Let

$$
s=\tau+i \theta
$$

The real and imaginary parts of $G(s)$ are

$$
\begin{aligned}
& \tau+a e^{-\tau} \cos \theta=0, \\
& \theta-a e^{-\tau} \sin \theta=0 .
\end{aligned}
$$

We consider first the single root on the real axis, and then the off-axis roots.

Roots on the real axis. On the real axis, (3.4a) becomes

$$
\tau+a e^{-\tau}=0 .
$$

For general $a$, this equation has zero, one, or two roots according as the exponential curve $g(\tau)=a e^{-\tau}$ intersects the line $g(\tau)=-\tau$ in zero, one, or two points. Here, with $a=e^{-1}$, we have the tangent case, with the single root $\tau=-1$.

Roots off the real axis. The pair of equations (3.4) for the roots can be rearranged to

$$
\begin{aligned}
\tau & =-\theta \cot \theta, \\
\theta & = \pm \sqrt{a^{2} e^{-2 \tau}-\tau^{2}} .
\end{aligned}
$$

The roots are indicated in Fig. 5. Since they occur as complex conjugate pairs, only the upper half-plane is shown. The trigonometric function (3.6a), shown by the dashed lines in the figure, has an infinite number of branches. These are intersected by the nearly vertical curve (3.6b), the solid line in the figure (a branch of $(3.6 \mathrm{~b}$ ) which produces no roots is not shown). Only every other intersection is a root of (3.4), as indicated by the dots in the figure. There are an infinite number of roots, which is typical of differential-difference equations. Because they are all in the left half-plane, the system is stable. As $\theta$ increases, the trigonometric curves given by (3.6a) flatten out, and the vertical root spacing approaches $2 \pi$. If we number the roots in order of increasing $\theta$, with root 1 at $\theta=0$, then for large $n$ the $n$th root approaches

$$
\begin{aligned}
\theta & \rightarrow \frac{4 n-3}{2} \pi, \\
\tau & \rightarrow \log \frac{a}{\theta} .
\end{aligned}
$$

The first three roots are given in Table I.

Table I. Poles of the Transfer Function

\begin{tabular}{cccc}
\hline & & $\begin{array}{c}\text { Decay } \\
\text { Length }\end{array}$ & Period \\
\hline$\tau$ & $\theta$ & $\tau^{-1}$ & $2 \pi / \theta$ \\
\hline-1 & 0 & 1 & $\infty$ \\
-3.09 & 7.46 & 0.323 & 0.842 \\
-3.66 & 13.9 & 0.326 & 0.453 \\
\hline
\end{tabular}




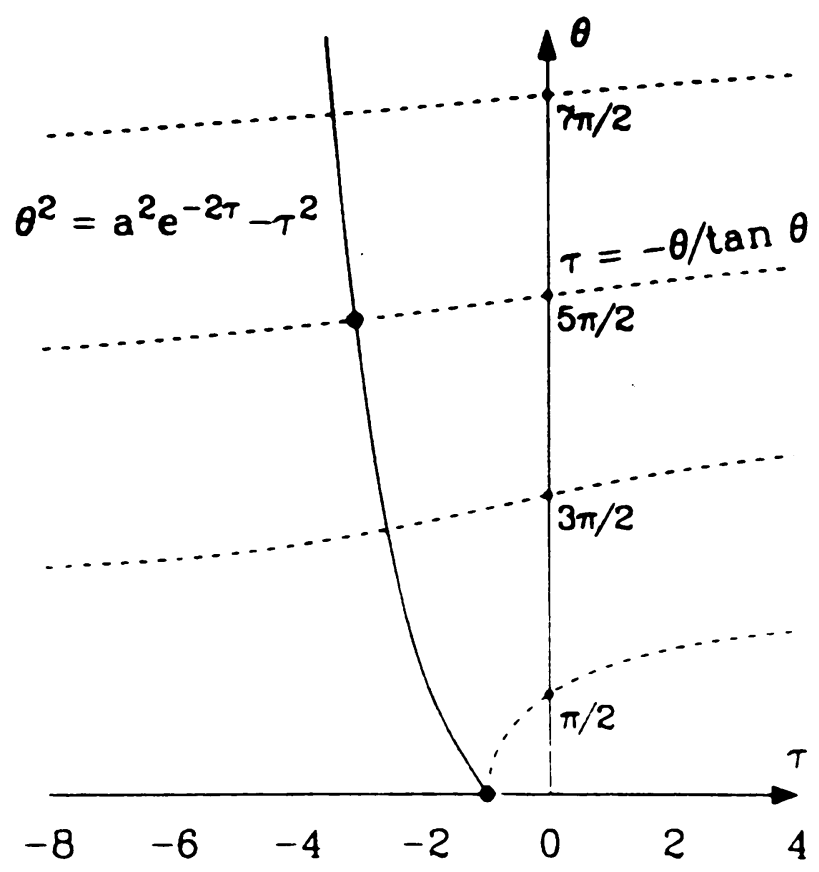

FIG. 5. Poles of the transfer function.

Eigenfunctions. The above roots are also the eigenvalues of the complete problem (1.1). For completeness, we set down here the corresponding eigenfunctions, although we will make use only of that for $s=-1$. Letting

$$
u(x, t)=y(x) e^{s t},
$$

and substituting in (1.1), we obtain for $y(x)$ the ordinary differential equation

$$
y^{\prime}(x)=-(s+1) y(x)+y(1) \text {. }
$$

Its solution, normalized to $y(1)=1$, is

$$
\begin{array}{ll}
s=-1: & y(x)=x \\
s \neq-1: & y(x)=\left(1-\frac{1}{s+1}\right) e^{(s+1)(1-x)}+\frac{1}{s+1} .
\end{array}
$$

IV. Solution of the differential-difference equation. We obtain a series solution of the differential-difference equation (2.10) for the initial-boundary condition (2.13) of Sec. II,

$$
u_{i}(x)=1, \quad u_{b}(t)=0,
$$

which generates the propagating discontinuity (2.14). The boundary condition $u_{b}(t)$ $=0$ makes the differential-difference equation (2.10) homogeneous. In region I, the initial condition $u_{i}(x)=1$ gives $u(x, t)=1$ and thus $u_{1}(t)=1$. From (2.14), we see that on the right boundary at $t=1$ (Fig. 4), the value of $u_{1}$ jumps from 1 down 
to $1-a$; this jump must be included in the initial data for $(2.10)$. With some new notation, (2.10) becomes (with this initial data)

$$
\begin{aligned}
\phi^{\prime}(t)+a \phi(t-1) & =0, \\
\phi(t) & =1, \quad 0 \leq t<1, \\
\phi(t) & =\alpha, \quad t=1,
\end{aligned}
$$

where we have defined

$$
\phi \equiv u_{1}, \quad \alpha \equiv 1-a .
$$

We seek the solution which is continuous $\left(C^{0}\right)$ for $t \geq 1$. Number the unit intervals as follows: let interval $n$ be that for which $n \leq t<n+1$, and let $\phi^{(n)}(t)$ be the solution in interval $n$. In interval zero $(0 \leq t<1)$ we have $\phi^{(0)}(t)=1$ from (4.2). The solution is

$$
\begin{array}{ll}
n=1: & \phi^{(1)}(t)=\alpha-a(t-1), \\
n>1: & \phi^{(n)}(t)=\sum_{i=0}^{n} c_{i}^{(n)}(t-n)^{i},
\end{array}
$$

where the coefficients $c_{i}$ are defined recursively by

$$
\begin{aligned}
c_{0}^{(n)} & =\sum_{i=0}^{n-1} c_{i}^{(n-1)}, \\
c_{i}^{(n)} & =-a \frac{c_{i-1}^{(n-1)}}{i}, \quad i=1,2, \ldots
\end{aligned}
$$

The solution of (4.2) is shown in Fig. 6. In the form (3.3), the dominant term in the solution is $2 e^{-t}$, from the root on the real axis (Fig. 5). This term is shown as the dashed line in Fig. 6. Beyond $t=1$, it quickly becomes a very good approximation to the solution as the higher frequencies decay.

For initial-boundary conditions "complementary" to (4.1), that is $u_{i}=0, u_{b}=1$, we find the problem

$$
\begin{aligned}
\phi^{\prime}(t)+a \phi(t-1) & =a, \\
\phi(t) & =0, \quad 0 \leq t<1, \\
\phi(t) & =1-\alpha, \quad t=1 .
\end{aligned}
$$

The solution is the "complement" of that of (4.2). That is, if the solution of (4.2) is $\hat{\phi}(t)$, then the solution of $(4.5)$ is $\phi(t)=1-\hat{\phi}(t)$.

V. Particular solutions. We present four simple particular solutions to the complete problem, two exact and two approximate, which give a good picture of the properties of the system (1.1). We name each by its input function.

1. Step. By inspection, we see that

$$
\begin{aligned}
u(x, t) & =1, \\
u_{i}(x) & =1, \\
u_{b}(t) & =1
\end{aligned}
$$




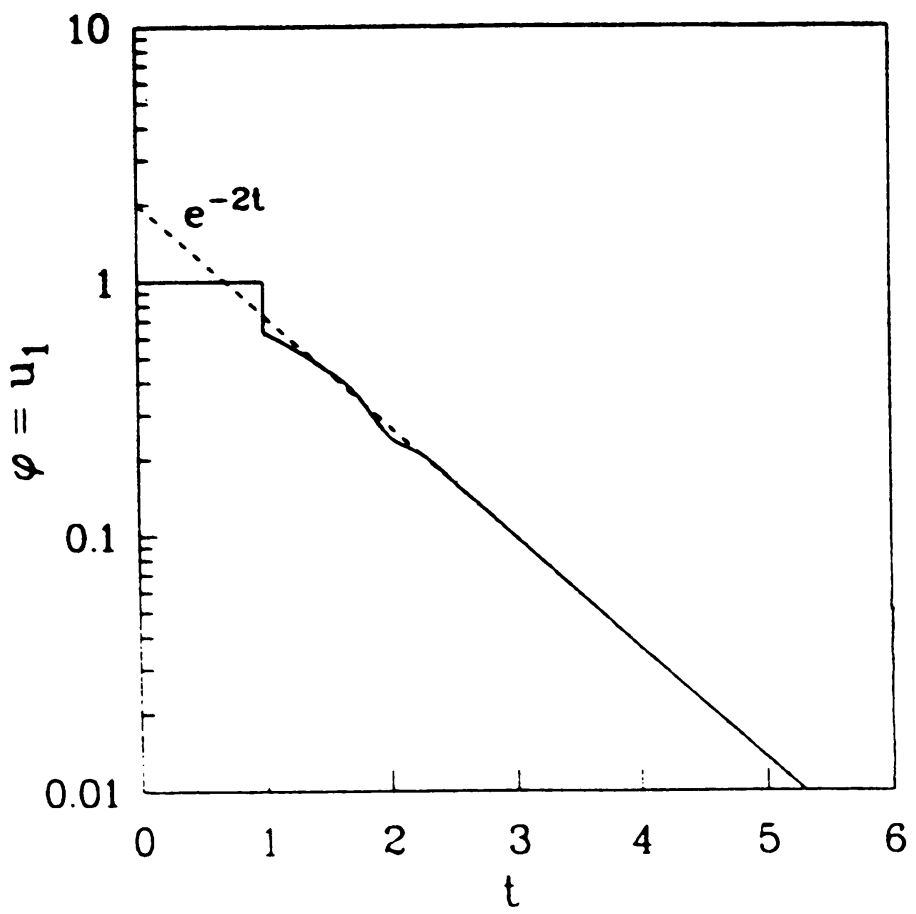

FIG. 6. Solution of the right-boundary differential-difference equation (25) for square-wave input.

is a solution of $(1.1)$.

2. Triangle. Another simple exact solution is

$$
\begin{aligned}
u(x, t) & =x e^{-t}, \\
u_{i}(x) & =x, \\
u_{b}(t) & =0 .
\end{aligned}
$$

This is the "fundamental" mode, the one in which only the "dominant" root-that on the real axis (Fig. 5)-is excited.

3. Square. If we take for $u_{1}(t)$ the approximate solution $2 e^{-t}$ of (4.2) for $t \geq 1$ (see Fig. 6), we find from (2.8) the approximate solution

$$
\begin{aligned}
u(x, t) & \cong 2 x e^{-t}, \quad t \geq 1, \\
u_{i}(x) & =1 \\
u_{b}(t) & =0 .
\end{aligned}
$$

4. Delayed step. This is the "complement" of the preceding solution, that is, the solution of $(4.5)$. It is

$$
\begin{aligned}
u(x, t) & \cong 1-2 x e^{-t}, \quad t \geq 1 \\
u_{i}(x) & =0 \\
u_{b}(t) & =1
\end{aligned}
$$


VI. Results. A helpful way of thinking about this problem is to relate the results conceptually to the standard ordinary-differential-equation problem of a linear system, such as a two-port electrical network, which produces an output signal (amplitude vs. time) from a given input signal (see any standard text, for example [6]). We extract such a problem from our system as follows. On a specified path in the $x-t$ space, $u(x, t)$ is a function of a single variable, such as arc length along the path. We pick two particular paths, one for the input function and one for the output function, and define a "time" variable $\tau$ as arc length on each. The obvious choice for the input path is the arc containing the given initial and boundary data, that is, the lower and left boundaries. For the input function we take the concatenation of the inital function $u_{i}(t)$ and the boundary function $u_{b}(t)$, and define $\tau$ as arc length on this path, beginning with $\tau=0$ at $x=1$ on the $x$-axis, proceeding leftward to the origin, and then upward along the $t$-axis. Thus our input function $u_{\text {in }}(\tau)$ is

$$
\begin{array}{llll}
u_{\text {in }}(\tau)=u_{i}(x), & 0 \leq \tau<1, & \tau=1-x, & \text { on } t=0, \\
u_{\text {in }}(\tau)=u_{b}(\tau), & \tau>1, & \tau=t+1, & \text { on } x=0 .
\end{array}
$$

For the output function we have more freedom of choice; we choose the path of greatest interest, the right boundary $x=1$, and take the output function to be $u_{1}(t)$,

$$
u_{\text {out }}(\tau)=u_{1}(t), \quad \tau=t, \quad \text { on } x=1 .
$$

In Table II we present the four solutions of the previous section in this framework. For each, we show the input function $u_{\text {in }}(\tau)$, the output function $u_{1}(t)$, and the complete solution $u(x, t)$. Case (1) is the constant solution produced by a step-function input. Case (2) is the fundamental solution, in which only the root on the real axis in Fig. 5 is excited. It is produced by the descending ramp (triangle) input shown. Case (3), an incomplete square wave followed by exponential decay, is produced by a square-wave input. The decaying part of this solution is twice the fundamental solution. Case (4), the "complement" of case (3), is produced by a delayed step input.

To summarize, we have studied the model equation (1.1) constructed to represent the behavior of perturbations on a steady detonation. Its distinguishing feature is the appearance of the unknown values of the dependent variable $u$ on one boundary as a source term. This gives rise to the differential-difference equation (2.10) for the values of $u$ on this boundary. From this equation we obtain the eigenvalues of the problem. We cast the problem into the form of a conventional linear ordinarydifferential-equation system with the applied perturbation as input and the shock perturbation as output. Both this output and complete solutions for the flow field for some simple input waveforms are given in Table II. The transfer function for the system has an infinite number of poles (the eigenvalues of the differential-difference equation) shown in Fig. 5. The lowest one (that on the real axis) is the dominant term in the solution. The others, when excited, appear as rapidly decaying transients.

Acknowledgment. I would like to thank John Bdzil of this laboratory for helpful conversations and a careful reading of the manuscript, and the reviewer for calling my attention to the work described in [3] and [4]. 


2. Triangle

\section{TABLE II. SOLUTIONS}

\section{REFERENCES}

[1] W. Fickett and W. C. Davis, Detonation, Chapt. 2, University of California Press, Berkeley, 1979

[2] W. Fickett, Decay of small planar perturbations on a strong steady detonation: A simple differentialdifference equation for the shock, Physics of Fluids 30, 1299-1309 (1987)

[3] R. K. Brayton, Nonlinear oscillations in a distributed network, Quart. Appl. Math 24, 280-301 (1967)

[4] K. L. Cooke and D. Krumme, Differential-difference equations and nonlinear initial-boundary value problems for linear hyperbolic partial differential equations, J. Math. Anal. Appl. 24, 372-387 (1968)

[5] R. Bellman and K. L. Cooke, Differential-difference equations, Academic Press, New York, 1963

[6] D. K. Frederick and A. B. Carlson, Linear systems in communication and control, Wiley, New York, 1971 